\title{
«Viele Ärzte machen Patienten krank»
}

\section{Daniel Lüthi}

Freier Journalist, Fotograf, Medientrainer, Bern

Sein Händedruck ist so stark, dass einer seiner ersten Sätze fast schicksalhafte Bedeutung erlangt hätte: «Wir behandeln hier alles - ausser Knochenbrüchen. Dafür fehlt bei uns allein ein Röntgenapparat.» Johann Schmid ist ein stämmiger, kräftiger Mann mit funkelnden Augen, kein verschrobenes Kräutermännchen, wie Vorurteile hätten vermuten lassen können. Einst war er Automechaniker und Lokomotivführer. Jetzt ist er vom «kant. appr. Naturarzt» - als einer der ersten, wie er stolz bemerkt - zum «eidg. dipl. Naturheilpraktiker (HFP)» und Prüfungsexperte geworden.

\section{Verschiedene Welten}

Im Wartezimmer hängt ein Bild aus exotischem Stein, das ihm der jüngere Bruder zum Praxisjubiläum geschenkt hat. Er arbeitet in Brasilien für eine grosse Schweizer Pharmafirma. «Diskussionen mit ihm offenbaren zwei völlig verschiedene Welten", sagt Johann Schmid. Auf der einen Seite: die Schulmedizin mit synthetischen und symptombezogenen Medikamenten, die der Bruder in Brasilien vertritt. Auf der anderen Seite: der Naturarzt in den Hügeln des Appenzellerlan-

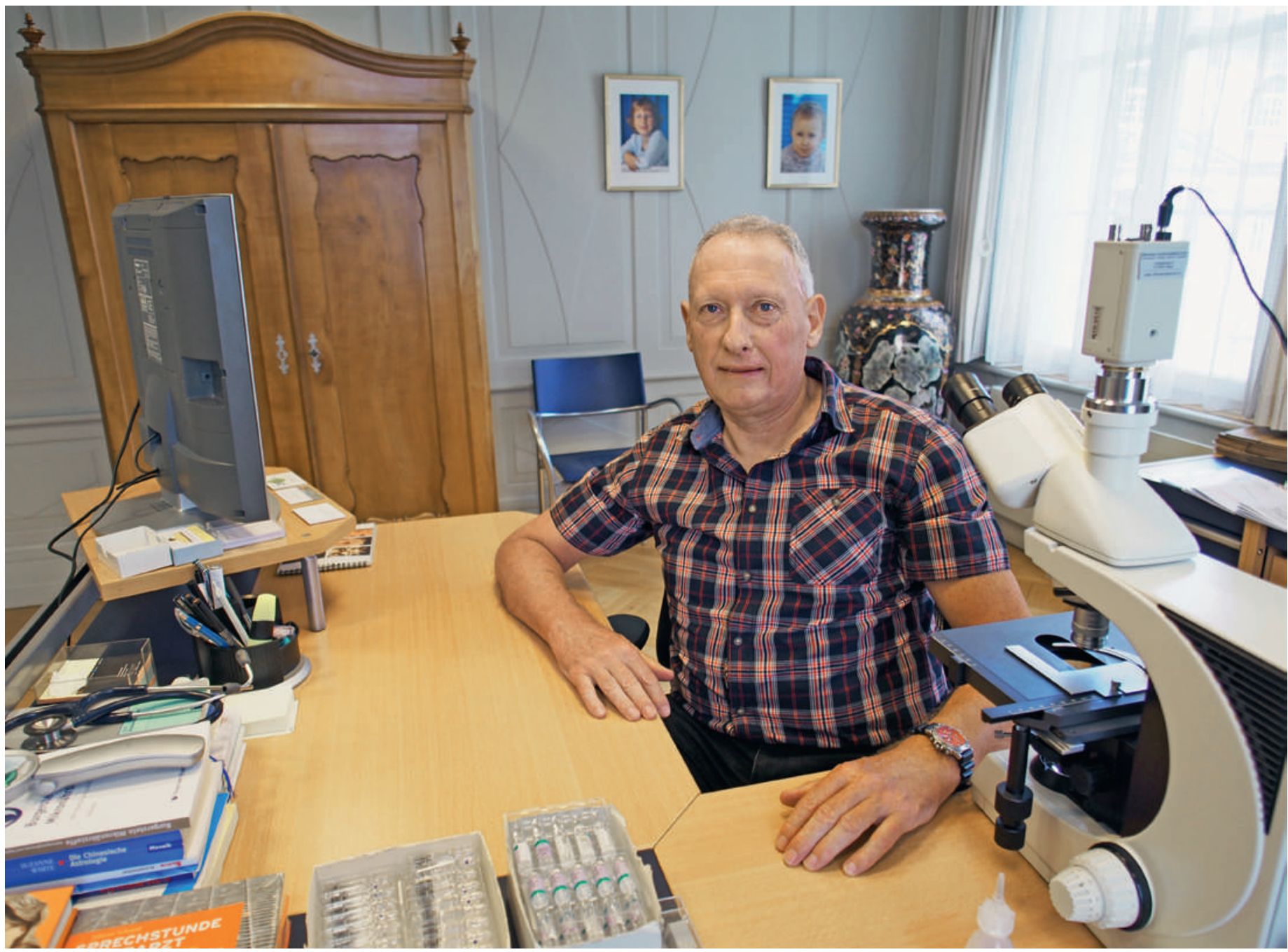


des, der sich, wie er sagt, für eine "ganzheitliche biologische Medizin» einsetzt.

«Die Bevölkerung wird zum Spielball der Industrie», schreibt er in seinem Buch Sprechstunde Naturarzt [1]. «Es wird alles der Profitmaximierung und der Markterweiterung untergeordnet.» Eines der Hauptprobleme sei, «dass die Medizin und die Pharmaindustrie nur mit kranken Menschen Geschäfte machen können. Wieso sich also das gute Geschäft vermiesen, indem man aus Kranken Gesunde macht?»

\section{«Ich will meine Patienten dafür gewinnen, dass sie selber gut zu ihrer Gesundheit schauen.»}

Das «Praxis-Institut Johann Schmid GmbH» liegt an prominenter Lage am Kirchplatz, im ersten Stock eines repräsentativen Gebäudes mit einer Grossbank im Erdgeschoss. Es umfasst, inklusive Seminarraum, 350 Quadratmeter und beschäftigt insgesamt zehn Personen. Er habe sehr viel Arbeit, sagt Schmid, und es gehe ihm, auch wirtschaftlich, gut. Der Mercedes vor dem Haus und seine Harley Davidson seien Symbole dafür. Aber Profitmaximierung sei nicht sein Ding. Eigentlich arbeite er sogar gegen die eigenen wirtschaftlichen Interessen, indem er die Prävention in den Vordergrund stelle: «Ich will meine Patienten dafür gewinnen, dass sie selber gut zu ihrer Gesundheit schauen und gar nicht erst krank werden.» Darum halte er auch viele Vorträge und schreibe er Bücher. «Aufklärungsarbeit», ergänzt er, und seine Augen funkeln besonders stark.

In einer Ecke des Behandlungszimmers steht eine grosse chinesische Vase. «Pflanzen gingen hier kaputt, die haben es bei all diesen kranken Menschen nicht ausgehalten", erzählt Schmid, und dass der Buddha aus Burma, der in der Ecke gegenüber steht, eher ein Zufall sei als der Ausdruck eines spirituellen Hintergrundes. Auf dem Tisch liegen Ampullen mit pflanzlichen Wirkstoffen, die Schmid ab und zu spritzt. In der Mitte steht ein Mikroskop, das für ihn zentral ist. Es ist ein Dunkelfeld-Mikroskop, mit dem er Blut untersucht. «Mit dieser Diagnosemöglichkeit kann ich das innere Milieu erkennen», erklärt er. Der Nussbaum-Schrank an der Wand ist ein Erbstück.

\section{Austherapierte therapieren}

«Früher ging es mir eher um die Pathogenese, also um die Entstehung und Entwicklung einer Krankheit», sagt Schmid. «Heute steht die Salutogenese im Vordergrund. Also die Frage, was der Einzelne tun kann, um gesund zu bleiben. Vier Begriffe sind zentral: Ernährung, Bewegung, Psycho-Hygiene und Umweltfakto-

\section{Johann Schmid}

Johann Schmid wurde 1953 in Amriswil geboren, wo er die Schulen besuchte und eine Lehre als Automechaniker absolvierte. Mit 20 kam er nach Zürich, wo er sich zum Lokomotivführer ausbilden liess. Zunehmend wurde er als Fahrdienstdisponent eingesetzt, was ihn mehr mit Menschen in Kontakt brachte und in ihm den Wunsch nach Veränderung

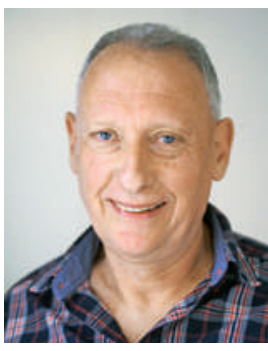
weckte. 1988 bis 1993 liess er sich in Zürich an der Deutschen Paracelsus-Schule - parallel zu seiner Tätigkeit bei den SBB zum Naturarzt ausbilden. Seit 1996 betreibt er in Heiden, AR eine eigene Praxis für biologische Ganzheitsmedizin. Johann Schmid ist Vater von vier Kindern. Mit dem jüngsten, seiner Partnerin und deren zwei Kindern lebt er in Rorschacherberg.

ren.» Dauerstress mit der Partnerin könne krank machen. Oder Elektrosmog. Wie auch immer: «Ich muss bei jeder Krankheit die Ursache finden. Wenn ich sie habe, kann ich den Patienten gesund machen.» Ein pensionierter Landwirt mit der Diagnose «Leukämie» sei zu ihm gekommen, erzählt er. Niemand habe ihn heilen können. Er habe herausgefunden, dass der Mann in seinem Leben sehr viele giftige Spritzmittel versprüht habe. «Etwa zwei Monate lang erhielt er von mir eine Entgiftungskur mit rein pflanzlichen Präparaten - und wurde gesund.» Bei einem 23-jährigen Patienten habe er die Leukämie auf eine Zahninfektion zurückführen können. Er habe den Zahnarzt angewiesen, dem Patienten alle betroffenen Zähne zu ziehen. So habe er auch ihn heilen können. Wunderheilung? «Keineswegs», antwortet Schmid umgehend. «Wunder produziert die Kirche da drüben, nicht wir.»

\section{«Wunder produziert die Kirche da drüben,} nicht wir.»

Tatsache jedoch sei, dass viele seiner Patientinnen und Patienten von einem Schulmediziner den Bescheid erhalten hätten, dass sie mit ihrer chronischen Krankheit zu leben hätten. "Zwei Drittel von denen, die zu uns kommen, sind von der Schulmedizin austherapiert. Davon machen wir zwei Drittel beschwerdefrei.»

\section{Mythos Milch}

Zurück zu dem, was krank macht. Entscheidend sei die Ernährung, sagt Schmid, «die ist für die Schulmedizin aber unbedeutend». Thunfisch zum Beispiel esse er nie, «der ist randvoll mit Quecksilber und Kadmium». Auch Schweinefleisch sei per se ungesund. Und, grosse Überraschung für einen Durchschnittsschweizer: «Kuh- 
milch ist gar nicht gut für uns Menschen. Wir sind doch keine Kälber!» Ganz vorne in seinem Buch geisselt Schmid den «Mythos vom Eiweissmangel». Das Gegenteil sei der Fall: Wir würden zu viel Eiweiss konsumieren und gerade deshalb krank. Aber eben: «Die Milchwirtschaft ist ein Milliardengeschäft, das mittels Werbung am Leben gehalten wird und sich gleichzeitig scheinbar um die Gesundheit der Bevölkerung bemüht.»

Auch Mediziner würden sich bisweilen nur scheinbar um die Gesundheit ihrer Patienten bemühen, sagt Schmid: «Viele Ärzte machen Patienten krank. Viele sind verlängerte Arme der Pharmaindustrie, geben unnötige oder gar schädliche Medikamente ab. Oder gibt es beispielsweise einen Grund, präventiv einen toxischen Cholesterin-Senker zu verordnen?»

Einer, der solches schreibt und sagt, muss viele Feinde haben, das scheint naheliegend. «Nun ja, man kennt mich überall», kommentiert Schmid, "und ich bin nicht gerade als Diplomat bekannt. So hatte ich schon ab und zu ein paar harte Diskussionen, als ich Medikamente absetzte, die ein Schulmediziner verschrieben hatte. Aber ich nehme halt kein Blatt vor den Mund. Und ich weiss, dass sie sich innerhalb ihres Systems nicht kritisieren. Aber viele schicken ihre Patienten zu mir, wenn sie nicht mehr weiter wissen.»

So auch Leute mit psychischen Leiden. «Rund die Hälfte der Menschen, die in psychiatrischen Kliniken sitzen, leiden an einem Mangel an Vitaminen, Mineralien und Spurenelementen. Auch bei einer Depression suche ich die Ursache. Das kann ein Hefepilz im Verdauungstrakt sein, der den Serotonin-Spiegel senkt. Oft muss jemand dann halt seinen Lebenswandel ändern.»

\section{Schulmedizin: ja, aber}

Johann Schmid betont, dass er nicht ein grundsätzlicher Gegner der Schulmedizin ist. «Sie hat durchaus auch ihre Berechtigung. Schwere akute Angelegenheiten kann ich nicht behandeln, dafür braucht's ein Antibiotikum oder ein Spital. Und manchmal ist auch eine Operation nötig, und da bewundere ich das Handwerk vieler Chirurgen.» Manchmal, aber nicht immer, ergänzt er. «Was ein anderer operiert hätte, habe ich auch schon weggespritzt.» Subkutan, mit rein pflanzli- chen Wirkstoffen. Eine Operation müsse immer ultima ratio sein, sagt Schmid, «eine Operation ist immer auch ein Eingeständnis, dass alles andere nicht gelungen ist."

Selber hat er sich übrigens auch schon unters Messer gelegt. «Ich hatte mal massive Kopfschmerzen. Sie kamen von einem zu hohen Augendruck. Die Medikamente des Augenarztes vertrug ich nicht, deshalb liess ich mich operieren.»

Und noch etwas betont Schmid: «Scharlatane gibt es überall, auch bei den Alternativmedizinern. Ein bisschen Handauflegen, ein wenig Hokuspokus reicht nicht. Ich mache nur, was ich - zum Beispiel biochemisch nachvollziehen und auch kontrollieren kann. Selber werde ich ja auch streng kontrolliert, vom Kanton und vom Berufsverband.»

\section{Leistung erbringen}

Seinen Patienten will Johann Schmid auch Vorbild sein. Er raucht nicht und trinkt nur wenig Alkohol, "am Wochenende eine Flasche Wein mit meiner Partnerin.» Süchtig ist er offenbar nur nach körperlicher Bewegung. Früher sei er viel Velo gefahren, erzählt er, zusätzlich habe er sich sechsmal pro Woche auch auf dem Hometrainer abgestrampelt, jetzt nur noch dreimal. Jeden Donnerstag gehe er im Alpsteingebiet wandern. Den Bieler 100-Kilometer-Lauf habe er mehrmals gemacht, leider habe er sein Ziel, ihn unter 10 Stunden zu schaffen, nicht erreicht. «Ich will überall eine gute Leistung erbringen", sagt Johann Schmid. Auch als Naturarzt in seiner Praxis. Das heisse für ihn allerdings nicht, dass er so viele Patienten wie möglich abfertigen wolle. «Mit meinem Team, darunter vier Naturärztinnen, empfange ich etwa 25 Patienten pro Tag. Bei anderen Ärzten können es 40 bis 50 sein. Aber eben: Die haben dann halt keine Zeit mehr für ein Gespräch, mit dem sie den Dingen auf den Grund kommen könnten.»

1 Schmid J. Sprechstunde Naturarzt. Schwellbrunn: Appenzeller Verlag; 2008.

Die nächste "Begegnung mit...»

Am Ende jeden Monats stellt die Schweizerische Ärztezeitung eine Persönlichkeit vor, die sich im Gesundheitswesen engagiert. Im Juli schildert Daniel Lüthi seine Begegnung mit Franco Denti, Hausarzt in Barbengo und Standespolitiker. 